\title{
Quantitative Evaluation of Metallographic Preparation Quality using EBSD
}

Philippe T. Pinard*, Pierre Hovington**, Marin Lagacé**, Gabriel M. Lucas***, George F. Vander Voort***, Raynald Gauvin*

* Materials and Mining Eng. Dept., McGill University, 3610 University St., Montreal, Quebec, Canada H3A 2B2

** Hydro-Quebec Research Institute, 1800 Lionel-Boulet Boul., Varennes, Quebec, Canada J3X 1S1

*** Buehler, Ltd., 41 Waukegan Road, Lake Bluff, IL 60044

In order to get a good polished surface, each step in the sample preparation routine must remove the surface damage left by the previous one. The surface deformation removal efficiency depends on the nature of the material and the sample preparation technique used. The amount of surface deformation can be quantified by measuring the depth of deformation.

In this study, the deformation produced by various cutting tools and by mechanical grinding will be characterized for the three main metallic crystal structures (FCC, BCC and HCP) using electron backscattered diffraction (EBSD). It has been shown that residual surface deformation has a detrimental effect on the quality of the EBSD diffraction patterns [1-4]. The dependence between the severity of deformation and the quality of the diffraction patterns makes EBSD an ideal technique to study the deformation left by various metallographic preparation techniques. The quality of the diffraction patterns can be measured using the signal-to-noise ratio of the Kikuchi bands. The quality indexes most commonly used to evaluate the quality of diffraction patterns are the pattern quality[3] and band contrast (HKL). Furthermore, local misorientation between adjacent points can reveal the level of strain induced by the deformation [5].

Large EBSD mappings were acquired on a Hitachi S-4700 cold-field emitter equipped with an HKL Nordlys II camera coupled to HKL's Channel 5 software. An accelerating voltage of $20 \mathrm{kV}$ with a beam current of $5 \mathrm{nA}$ along with a pattern binning of $8 \times 8$ were used to allow for fast pattern acquisition. Because of large differences in the damage depths between preparation techniques, different magnifications and step sizes were used. All the patterns were saved for further processing using the EBSD plugin found in RML-Image [6].

The large maps were divided into sections of equal width each containing more than 800 pixels in total (Figure 1). A gaussian distribution was fitted over the band contrast data for each section. The distribution averages are plotted as a function of the distance from the damaged surface. The results show an increase in the average band contrast from the damage surface towards to center of the sample until it eventually reaches a plateau corresponding to the average band contrast of the bulk sample. The $90 \%$ deformation point was calculated by fitting an error function (erf) to the results. Figures 2 and 3 show the distributions for two extreme cases of cutting deformation on titanium. The deformation is harder to evaluate from the mean angular deviation (MAD) and local misorientation maps because both values rely on the precise indexing of the Kikuchi bands and not only on their signal to noise ratio as the pattern quality and the band contrast does.

The pattern quality as well as other quality indexes as proposed by Wright and Nowell[3] will be evaluated. An algorithm to remove the effect of grain boundaries from the analysis will be implemented.

[1] A.J. Wilkinson and D.J. Dingley (1991), Acta Materialia, V. 39, pp. 3047-3055

[2] J. Wu, P.J. Wray, C.I. Garcia, M. Hua and A. J. Deardo (2005), ISIJ International, V. 45, pp. 254-262

[3] S.I. Wright and M.M. Nowell (2006), Microscopy and Microanalysis, V. 12, pp. 72-84

[4] J. Cocle, R. Gauvin and S. Yue (2008), Microscopy and Microanalysis, V. 14, pp. 916-917

[5] L.N. Brewer, M.A. Othon, L.M. Young and T.M. Angeliu (2006), Microscopy and Microanalysis, V. 12, pp.85-91

[6] M. Lagacé, RML-Image (www.rml-image.com). 


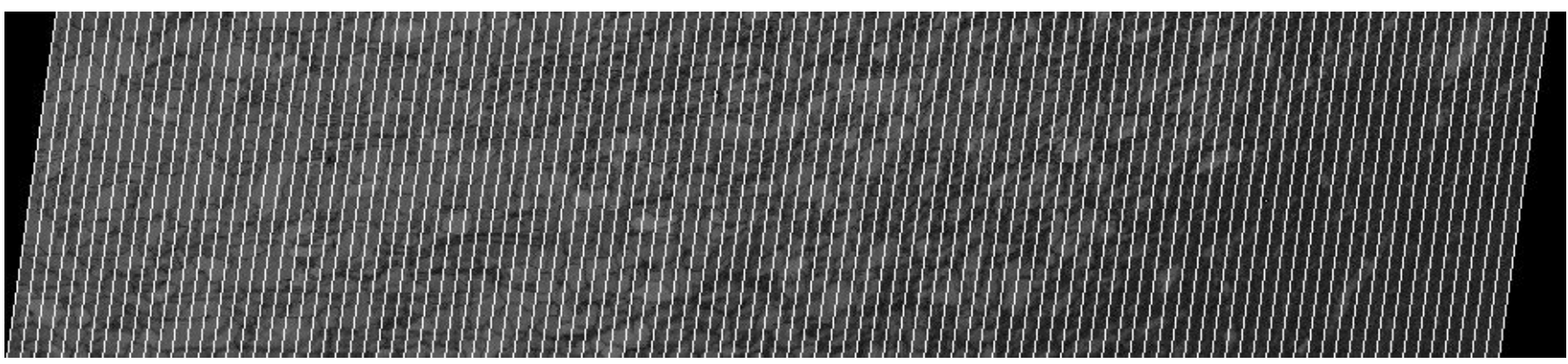

Fig. 1: Example of a sectioned band contrast map. The sections are parallel to the damage surface located on the left.

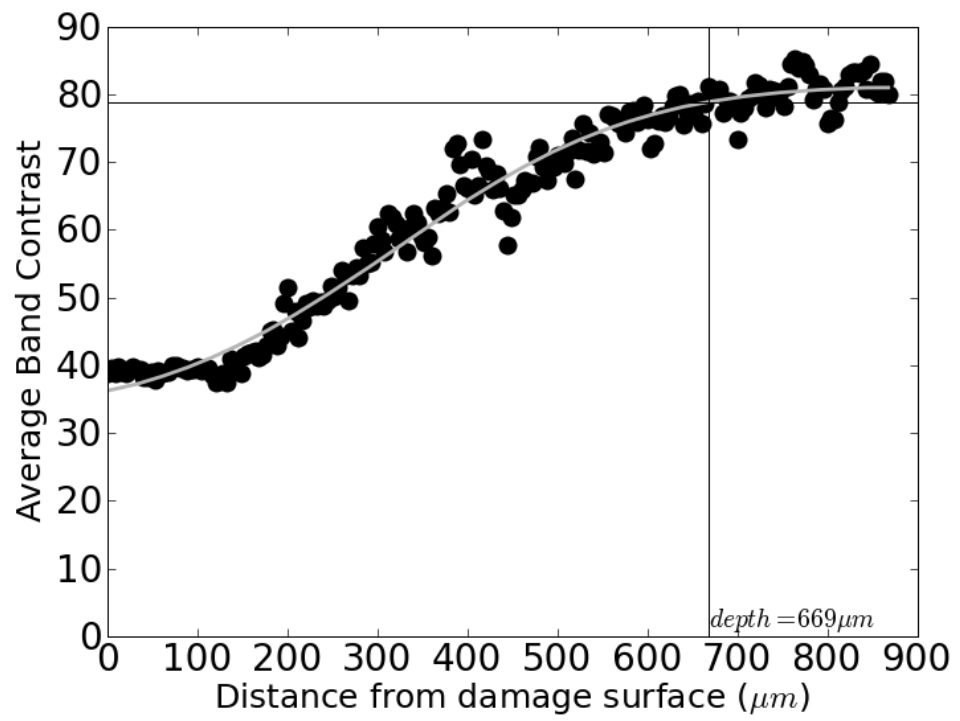

Fig. 2: Shear cut sample with a $90 \%$ deformation depth of $669 \mu \mathrm{m}$ measured using the average band contrast

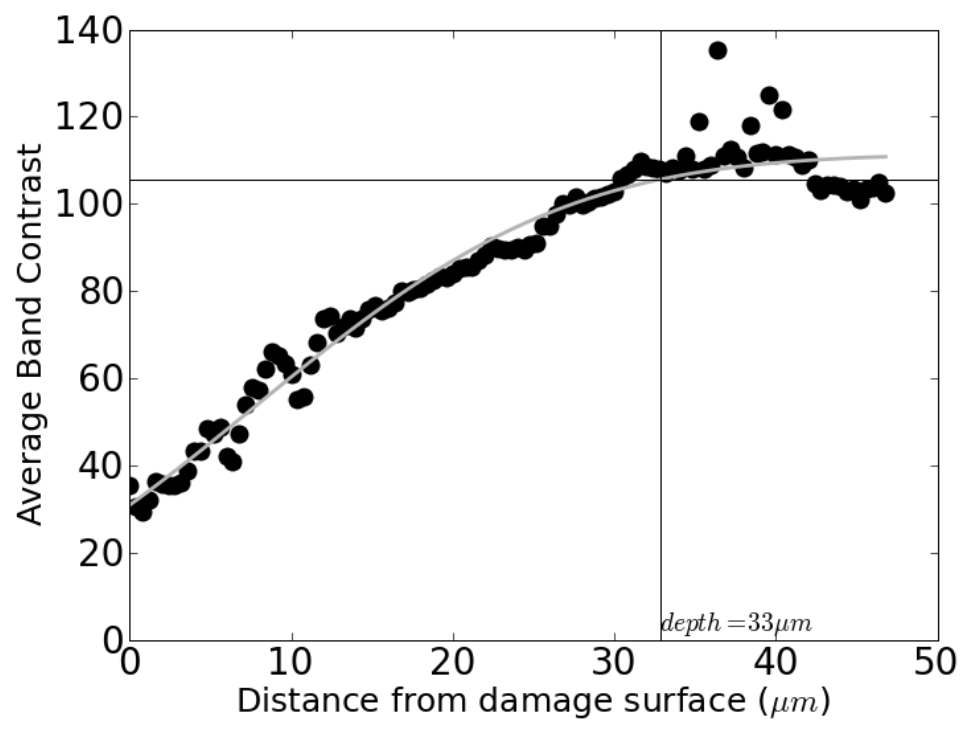

Fig. 3: Diamond saw cut sample with a $90 \%$ deformation depth of $33 \mu \mathrm{m}$ measured using the average band contrast 